$05,12,01$

\title{
Отклик решетки магнитных наночастиц на импульс магнитного поля вблизи границы устойчивости
}

\author{
(C) А.М. Шутый, Д.И. Семенцов \\ Ульяновский государственный университет, \\ Ульяновск, Россия \\ E-mail: shuty@mail.ru \\ (Поступила в Редакцию 28 июня 2017 г.)
}

Для плоских решеток магнитных наночастиц, обладающих кубической кристаллографической анизотропией, исследуется динамический отклик на импульс внешнего магнитного поля системы, находящейся вблизи границы устойчивого равновесия. Выявлены условия, при которых имеет место распространение колебаний магнитных моментов от отдельных диполей на всю систему, в результате чего реализуется отклик решетки, значительно больший по продолжительности внешнего импульса и с амплитудой относительно слабо зависящей от начальных условий и параметров внешнего поля. Рассмотрены также процессы, при которых действие импульса приводит к переориентации только отдельных диполей решетки.

Работа выполнена при поддержке Министерства образования и науки РФ в рамках Государственного задания № 3.6825.2017/ПЧ и проекта 14.Z50.31.0015.

DOI: 10.21883/FTT.2018.01.45294.208

\section{1. Введение}

В последние годы ансамбли магнитных наночастиц с различной топологией привлекают все большее внимание исследователей. Связано это с тем, что в динамическом поведении таких ансамблей проявляются не только свойства отдельных частиц, но и коллективные эффекты, определяемые типом взаимодействия наночастиц, их взаимным расположением, свойствами матрицы, в которой сформирован ансамбль [1-7]. Особый интерес представляют двумерные магнито-упорядоченные структуры, состоящие из дипольно взаимодействующих однодоменных наночастиц ферромагнитного металла. Дискретность указанных структур приводит к существенным отличиям их равновесных состояний и динамических режимов от свойств макроскопических монодоменных объектов. К таким отличиям, в частности, могут быть отнесены бистабильные состояния решеток, обусловленные наличием различных ориентационных конфигураций с неодинаковым суммарным магнитным моментом, а также возможных управляемых переходов между конфигурациями и динамических колебательных режимов магнитного момента системы при их перемагничивании [8-13].

Наряду с этим, регулярные ансамбли магнитных наночастиц различной размерности могут служить средой для сверхплотной записи и хранения информации. Возможность записи информации на решетке магнитных диполей основана на изменении равновесной конфигурации магнитных моментов за счет воздействия радиоимпульсов магнитного поля, а считывание обеспечивается возбуждением возникшей конфигурации маломощным радиоимпульсом на частоте ферромагнитного резонанса и сканированием частоты отклика дипольной системы [14-16].
В работе [17] исследована динамика квадратной решетки однодоменных наночастиц, обладающих магнитной кубической анизотропией (КА), на воздействие внешнего магнитного поля. При этом выявлены квазипериодические и хаотические режимы, а также особенности отклика различных по параметрам систем на действие импульса поля. В настоящей работе основное внимание уделено исследованию отклика данных дипольных решеток на импульс внешнего магнитного поля при параметрах структуры, отвечающих состоянию вблизи границы устойчивого равновесия. Рассмотрены процессы распространения хаотических колебаний от краевых диполей на всю систему, а также процессы переориентации отдельных диполей под действием внешнего импульса. Проводится сравнение отклика решетки диполей на импульс магнитного поля при различных параметрах как решетки, так и действующего импульса.

\section{2. Исходные уравнения}

Рассмотрим квадратную решетку из $10 \times 10$ взаимодействующих друг с другом сферических однодоменных наночастиц, которые представляются точечными диполями с одинаковым по величине магнитным моментом $\left|\mathbf{m}_{i}\right|=m$, пропорциональным объему наночастицы $V$. Энергию $i$-ого диполя запишем в виде суммы зеемановской энергии во внешнем магнитном поле $\mathbf{H}$, энергии КА наночастицы и энергии диполь-дипольного взаимодействия

$$
W_{i}=-\mathbf{m}_{i} \mathbf{H}+W_{a}\left(\mathbf{m}_{i}\right)+\sum_{n} W_{d}\left(\mathbf{m}_{i}, \mathbf{m}_{n}\right)
$$

Внешнее магнитное поле является суммой статического и высокочастотного полей $\mathbf{H}=\mathbf{H}_{c}+\mathbf{H}(t)$; энергия КА 
в случае, когда оси $X, Y, Z$ направлены вдоль трех кристаллографических осей $\langle 100\rangle$ наночастицы, имеет вид

$$
W_{a}=\frac{V K_{1}}{m^{4}}\left(m_{i x}^{2} m_{i y}^{2}+m_{i y}^{2} m_{i z}^{2}+m_{i z}^{2} m_{i x}^{2}\right),
$$

где $K_{1}$ - константа КА материала наночастицы. Будем считать ось $X$ перпендикулярной плоскости решетки, а две другие оси - параллельными ее сторонам. Энергию диполь-дипольного взаимодействия представим в виде

$$
W_{d}\left(\mathbf{m}_{i}\right)=\sum_{n \neq i}\left(\frac{\mathbf{m}_{i} \mathbf{m}_{n} r_{i n}^{2}-3\left(\mathbf{m}_{i} \mathbf{r}_{i n}\right)\left(\mathbf{m}_{n} \mathbf{r}_{i n}\right)}{r_{i n}^{5}}\right),
$$

где $\mathbf{r}_{i n}$ и $r_{i n}-$ радиус-вектор и расстояние между $i$-ым и $n$-ым диполями.

Динамика каждого из магнитных моментов описывается уравнением Ландау-Лифшица с релаксационным членом в форме Гильберта [18]

$$
\frac{\partial \mathbf{m}_{i}}{\partial t}=-\gamma \mathbf{m}_{i} \cdot \mathbf{H}_{i}^{\mathrm{ef}}-\frac{\alpha}{m_{i}} \mathbf{m}_{i} \cdot \frac{\partial \mathbf{m}_{i}}{\partial t}
$$

где $\gamma$ - гиромагнитное отношение, $\alpha$-одинаковый для всех частиц параметр диссипации. Эффективное магнитное поле, создаваемое в месте расположения $i$-го диполя остальными диполями и внешним полем $\mathbf{H}$, имеет вид

$$
\mathbf{H}_{i}^{\mathrm{ef}}=-\frac{\partial W_{i}}{\partial \mathbf{m}_{i}}=\mathbf{H}+\mathbf{H}_{a i}+\sum_{n \neq i} \frac{3\left(\mathbf{m}_{n} \mathbf{r}_{i n}\right) \mathbf{r}_{i n}-\mathbf{m}_{n} r_{i n}^{2}}{r_{i n}^{5}} .
$$

Каждая из трех компонент $(v \equiv x, y, z)$ поля КА определяется выражением

$$
H_{a i v}=-\frac{2 V K_{1}}{m} \mu_{i v}\left(1-\mu_{i v}^{2}\right),
$$

где $\boldsymbol{\mu}_{i}=\mathbf{m}_{i} / m$. Далее перейдем к безразмерным параметрам: $\mathbf{e}_{i n}=\mathbf{r}_{i n} / r_{i n}, \tau=\left(m \gamma / d^{3}\right) t$, где $d$ - диаметр наночастиц; $l_{i n}=r_{i n} / d$. Примем, что расстояние между центрами ближайших диполей системы одинаково и равно $a$. В безразмерных параметрах уравнения (5) принимают вид

$$
\frac{\partial \boldsymbol{\mu}_{i}}{\partial \tau}=-\boldsymbol{\mu}_{i} \cdot \mathbf{h}_{i}^{\text {ef }}-\alpha \boldsymbol{\mu}_{i} \cdot \frac{\partial \boldsymbol{\mu}_{i}}{\partial \tau},
$$

где

$$
\mathbf{h}_{i}^{\mathrm{ef}}=\mathbf{h}+\mathbf{h}_{a i}+\sum_{n \neq i}\left[\frac{3\left(\boldsymbol{\mu}_{n} \mathbf{e}_{i n}\right) \mathbf{e}_{i n}-\boldsymbol{\mu}_{n}}{l_{i n}^{3}}\right] .
$$

При этом внешнее поле $-\mathbf{h}=\mathbf{H} d^{3} / m$, а поле КА

$$
h_{a i j}=-2 k_{1} \mu_{i j}\left(1-\mu_{i j}^{2}\right) \text {, }
$$

где $k_{1}=K_{1} V d^{3} / m^{2}-$ константа анизотропии. Для дальнейшего анализа векторное уравнение (7) представляется тремя скалярными уравнениями. Так, для $X$-компоненты получаем

$$
\begin{aligned}
\left(1+\alpha^{2}\right) \frac{\partial \mu_{i x}}{\partial \tau} & =\left(\mu_{i z}+\alpha \mu_{i x} \mu_{i y}\right) h_{i y}^{\mathrm{ef}} \\
& -\left(\mu_{i y}-\alpha \mu_{i z} \mu_{i x}\right) h_{i z}^{\mathrm{ef}}-\alpha\left(1-\mu_{i x}^{2}\right) h_{i x}^{\mathrm{ef}}
\end{aligned}
$$

Уравнения для остальных компонент величины $\partial \boldsymbol{\mu}_{i} / \partial \tau$ имеют аналогичный вид и могут быть получены циклической перестановкой индексов $x, y, z$.

\section{3. Равновесные конфигурации}

Равновесные ориентации отдельных магнитных моментов квадратной решетки $10 \times 10$ и динамика суммарного магнитного момента решетки в целом определялись с учетом связи всех элементов ансамбля друг с другом на основе численного решения приведенных выше уравнений методом Рунге-Кутта четвертого порядка. В отсутствии внешних полей равновесные ориентационные конфигурации находятся заданием произвольных начальных состояний магнитных моментов всех диполей, из которых система приходит к стационарному состоянию согласно приведенным уравнениям движения.

В случае рассматриваемых здесь решеток с константой КА $k_{1}=-0.5$ (что соответствует наночастицам из атомов железа) в отсутствие внешнего статического поля при близком расположении наночастиц (параметр, определяющий расстояние между элементами $a / d<2)$, как правило, реализуются конфигурации с нулевым или близким к нулю суммарным магнитным моментом $\mathbf{M}=\sum \boldsymbol{\mu}_{i}$. При этом с расширением системы увеличивается число возможных равновесных конфигураций, отличающихся ориентацией отдельных диполей и величиной свободной энергии системы. В случае систем с $a / d \geq 2.7$ влияние КА становится преобладающим, и оказываются равновесными любые конфигурации, при которых магнитные моменты отдельных наночастиц ориентированы по направлению кристаллографических осей. Так как нами рассматривается анизотропия типа [100], направление ориентации магнитных моментов наночастиц приближается к осям системы $X, Y$ или $Z$.

На рис. 1 приведены равновесные конфигурации дипольной решетки с параметром $a / d=3,1.5,1$ (конфигурации $1-3)$, полученные после достаточно продолжительного действия на нее (и последующего выключения) статического поля, направленного вдоль оси $Y$. Видно, что в первом случае все диполи остались ориентированными по оси $Y$. Во втором случае основная часть диполей решетки разбилась на цепочки, ориентированные либо в положительном, либо в отрицательном направлении оси $Y$ (лишь малая часть диполей вблизи края решетки имеет ориентацию вдоль оси $Z$ ). В случае, когда наночастицы располагаются наиболее близко друг к другу, основная часть диполей разбилась на цепочки, ориентированные по оси $Z$.

На рис. 2, а приведена зависимость энергии дипольдипольного взаимодействия от нормированного расстояния между элементами для трех различных конфигураций: все диполи ориентированы перпендикулярно плоскости системы (кривая 1), диполи лежат в плоскости системы и ориентированы вдоль оси $Y$ (кри- 


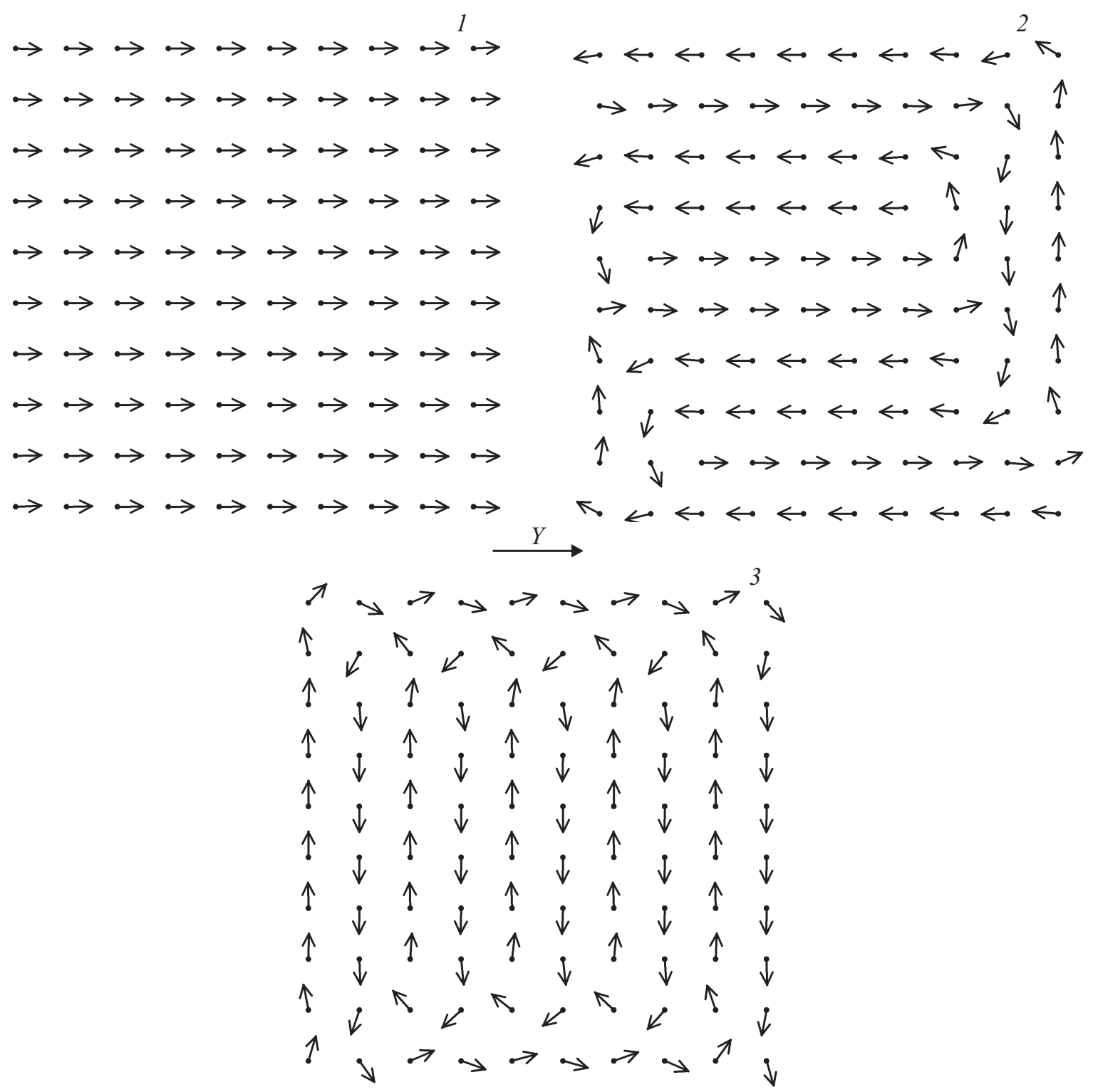

Рис. 1. Равновесные конфигурации дипольной решетки с параметром $a / d=3,1.5,1$ (конфигурации $1-3$ ), полученные после продолжительного действия на нее (и последующего выключения) статического поля, направленного вдоль оси $Y$; константой КА $k_{1}=-0.5$.

вая 2), диполи лежат в плоскости и разбиты на ряды, чередующиеся по направлению $\pm Y$ (кривая 3). Видно, что конфигурацией с наименьшей энергией дипольдипольного взаимодействия является конфигурация с чередованием противоположно ориентированных рядов, a с наибольшей - конфигурация с перпендикулярным направлением диполей. Однако разница между данными энергиями сильно уменьшается с увеличением расстояния между элементами системы. Поэтому в результате действия кубической анизотропии при увеличении параметра $a / d$ вначале становится устойчивой конфигурация с ориентацией всех диполей по оси $Y$, а затем (при больших $a / d$ ) по перпендикулярной оси $X$.

Ни рис. 2, $b$ приведена зависимость энергии рассматриваемой решетки (при отсутствии внешнего поля) от полярного угла ориентации магнитных моментов диполей, отсчитываемого от оси $X$. Отсчитываемый от оси $Y$ азимутальный угол принимается равным нулю. Расстояние между диполями $a / d=4,3,2.5,2$ (кривые $1-4)$. Видно, что при $a / d<2.5$ устойчивой оказывается только плоскостная конфигурация. В случае же больших расстояний между элементами решетки наблюдается энергетический минимум и при ориентации всех диполей перпендикулярно плоскости системы, причем с отдалением диполей друг от друга потенциальная яма увеличивается, так как энергия диполь-дипольного взаимодействия падает, а энергия КА остается неизменной.

На рис. 2, с приведена аналогичная зависимость, но лишь для одного углового диполя (непрерывные кривые) и одного из внутренних, ближайших к центру 

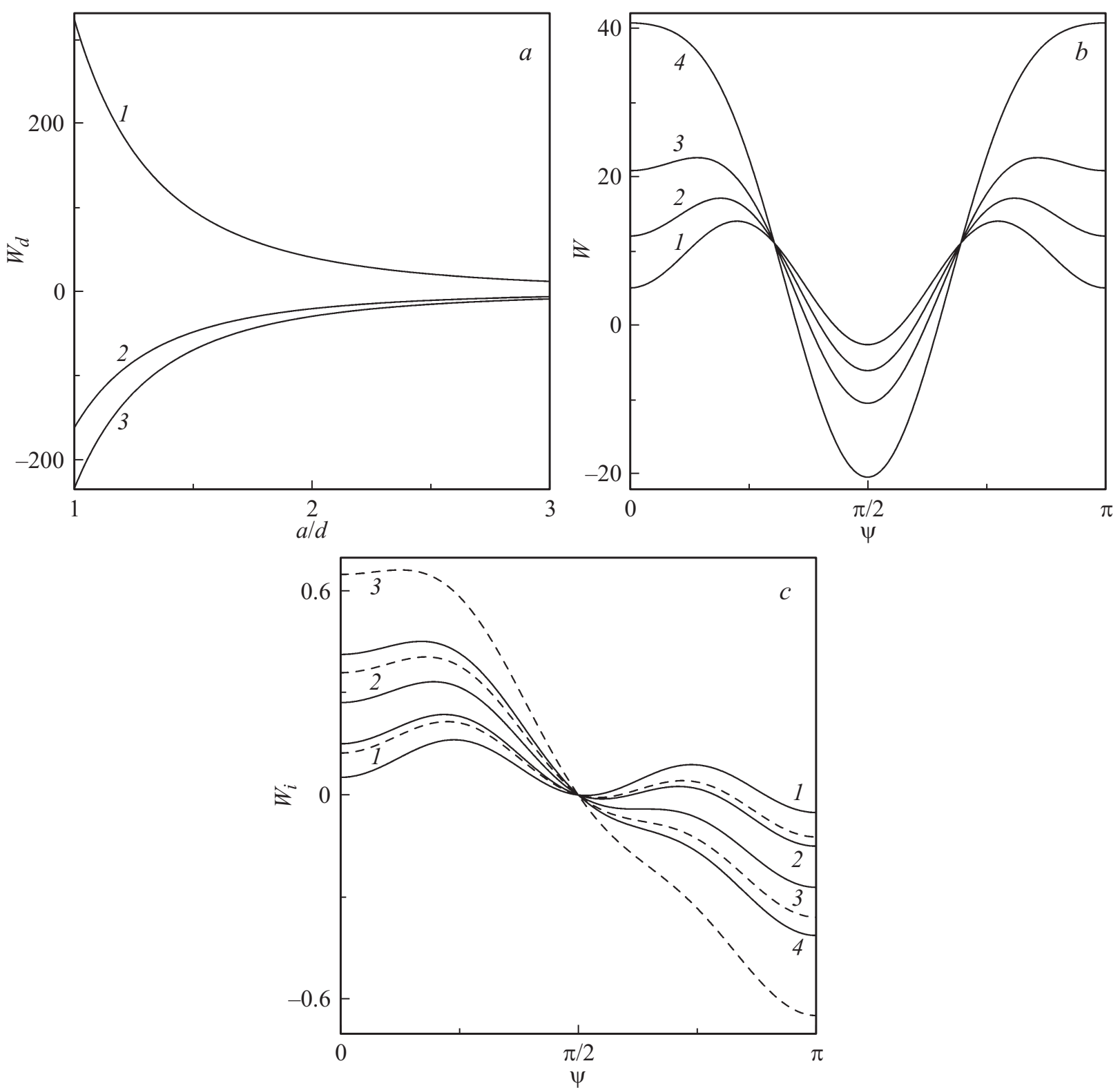

Рис. 2. Зависимость энергии диполь-дипольного взаимодействия решетки от нормированного расстояния между диполями (a) для конфигураций: все диполи ориентированы по $X$ (кривая 1), по $Y$ (кривая 2), диполи разбиты на ряды, чередующиеся по направлению $\pm Y$ (кривая 3). Зависимость энергии решетки от полярного угла $\psi$ ориентации магнитных моментов $(b)$ при $a / d=4$, $3,2.5,2$ (кривые 1-4). Зависимость от $\psi$ энергии одного углового диполя (непрерывные кривые) и одного ближайшего к центру системы диполей (пунктирные кривые), когда остальные диполи ориентированы по оси $X(c)$, при $a / d=4,2.8,2.3,2$ (кривые $1-4$ ).

системы, диполей (пунктирные кривые), при условии, что все остальные диполи ориентированы перпендикулярно плоскости решетки. Расстояние между диполями $a / d=4,2.8,2.3,2$ (кривые $1-4$ ). Видно, что при $a / d \geq 2.8$ устойчивой оказывается конфигурация, когда только один из диполей лежит в плоскости системы. При более близком расположении элементов в решетке данная конфигурация неустойчива, и выделенный диполь будет стремиться ориентироваться в направлении остальных диполей.

Как показывают более точные численные расчеты, в диапазоне значений параметра $2.7 \leq a / d \leq 2.8$ имеет место слабо выраженный минимум потенциальной энергии решетки с перпендикулярной плоскости системы конфигурацией. При этом также имеет место устойчивое равновесие одного из диполей (преимущественно углового) в плоскости системы, когда остальные диполи ориентированы перпендикулярно. В результате этого указанное равновесное состояние оказывается чувствительным к слабым внешним воздействиям, что может быть использовано для усиления отклика системы на внешний сигнал. Заметим, что при учете тепловых колебаний равновесные конфигурации со слабо выраженным энергетическим минимумом оказываются метастабиль- 

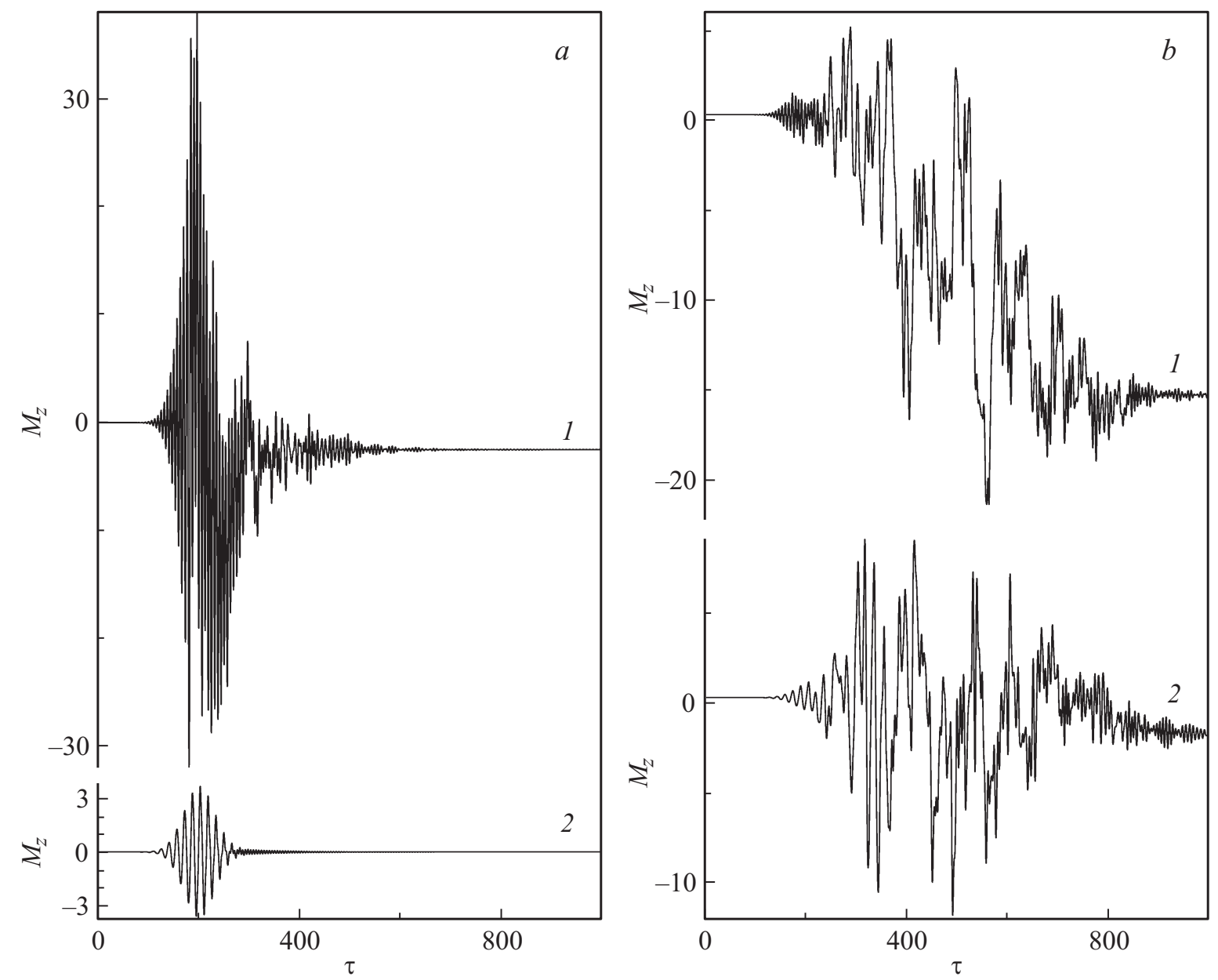

Рис. 3. Зависимость от времени $Z$-компоненты суммарного магнитного момента при действии импульса поля, линейно поляризованного по оси $X$ при частоте $\Omega=1,0.4$ (кривые 1 и 2) и параметрах $\tau_{0}=30, h_{0 x}=0.1 ; \alpha=0.01$. В исходной конфигурации все диполи ориентированы по оси $Y$ при $a / d=3(a)$ или по оси $X$, за исключением четырех угловых диполей (подсистема $2 \times 2$ ), ориентированных по оси $Y$, при $a / d=2.7(b)$.

ными, и интересующий нас диапазон параметра решетки $a / d$ окажется смещенным в область больших значений.

\section{4. Отклик системы на импульс}

Рассмотрим отклик системы при различных параметрах на импульс вешнего магнитного поля, который имеет гауссову временную огибающую

$$
\mathbf{h}(\tau)=\mathbf{h}_{0} \sin (\Omega \tau) \exp \left(-\tau^{2} / 2 \tau_{0}^{2}\right),
$$

где $\tau_{0}$ - длительность импульса, а $\Omega-$ безразмерная несущая частота, связанная с реальной несущей частотой соотношением $\Omega=\left(d^{3} / m \gamma\right) \omega$. Заметим, что резонансная частота изолированных магнитных моментов $\omega_{0}=\gamma H$, и

$$
\Omega_{0}=\frac{d^{3} \omega_{0}}{m \gamma}=h
$$

Взаимодействие между диполями изменяет частоту резонанса, при этом зависимость амплитуды прецессии от частоты поля становится нелинейной. При отсутствии взаимодействия между диполями резонансная частота определялась бы только полем анизотропии $\Omega_{r}=2\left|k_{1}\right|$.

На рис. 3 приведена зависимость от времени $Z$-компоненты суммарного магнитного момента системы при действии импульса внешнего поля, линейно поляризованного вдоль перпендикулярного к решетке направления, при частоте импульса $\Omega=1,0.4$ (кривые 1 и 2) и его параметрах $\tau_{0}=30, h_{0 x}=0.1$. Параметр диссипации здесь и далее принят равным $\alpha=0.01$. В случае $(a)$ исходной является однородная намагниченность решетки по оси $Y$, при параметре $a / d=3$. В случае же $(b)$ в исходной конфигурации все диполи, кроме четырех угловых (подсистема $2 \times 2$ ), ориентированы перпендикулярно системе, т.е. вдоль оси $X$, а четыре указанных угловых диполя решетки ориентированы вдоль оси $Y$; при этом параметр решетки $a / d=2.7$. Так как рассматривается система с константой КА $k_{1}=-0.5$, частота $\Omega=1$ близка к резонансной. При данной частоте в случае $(a)$ импульс действует на всю систему с лежащими в ее плоскости диполями, что приводит к большой амплитуде отклика системы. Однако при удалении частоты от 

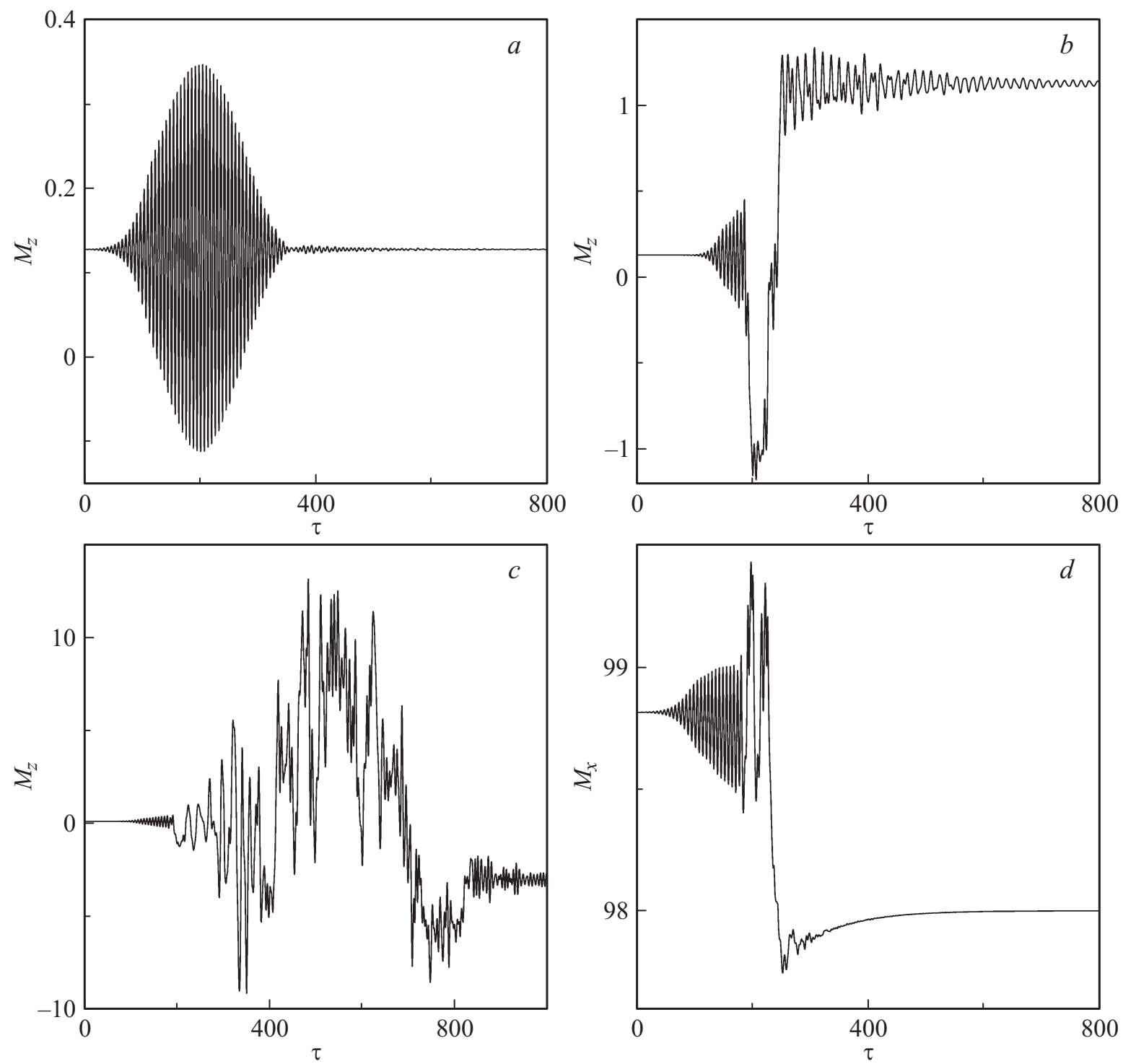

Рис. 4. Зависимость от времени компонент суммарного магнитного момента для системы с $a / d=2.7$ и исходной конфигурацией с одним угловым диполем, ориентированным по оси $Y$ (остальные диполи ориентированы по оси $X$ ), при действии импульса с параметрами $\Omega=1, \tau_{0}=50(a, d), \tau_{0}=30,40(c, d), h_{0 x}=0.1(a)$ и $h_{0 x}=0.2(b-d)$.

резонансного значения амплитуда динамики решетки резко падает (а, кривая 2), сами же колебания - в отличие от первого значения частоты - приобретают регулярный характер.

Иная ситуация имеет место в случае $(b)$. Здесь импульс внешнего поля эффективно действует только на четыре угловых диполя. Так как при значении $a / d=2.7$ решетка близка к неустойчивому ориентационному состоянию, возникающая динамика угловых диполей приводит к колебаниям соседних с ними диполей. Фронт хаотических колебаний со временем распространяется на всю систему. В результате отклик системы на импульсное воздействие оказывается более продолжительным, а его амплитуда в некотором диапазоне частоты слабо зависит от ее значений. Амплитуда отклика в этом случае меньше амплитуды отклика конфигурации $(a)$ в условиях резонанса, однако в случае $(b)$ можно ограничиться локальным полем, действующим только на четыре угловых диполя и не затрагивающим всю систему.

Ситуация, аналогичная случаю рис. $3, b$, имеет место и при конфигурации, когда только один диполь лежит в плоскости решетки. Однако для осуществления колебаний всей системы при этом требуются большие по амплитуде импульсы поля, и реализация процесса оказывается более чувствительной к параметрам импульса, в частности к его продолжительности. На рис. 4 приведена зависимость от времени компонент суммарного магнитного момента для системы с параметром $a / d=2.7$ и исходной конфигурацией с одним угловым диполем, ориентированным по оси $Y$ (остальные диполи ориентированы по оси $X)$, при действии импульса с параметрами $\Omega=1, \tau_{0}=50(a, d), \tau_{0}=30,40(c, d)$, 


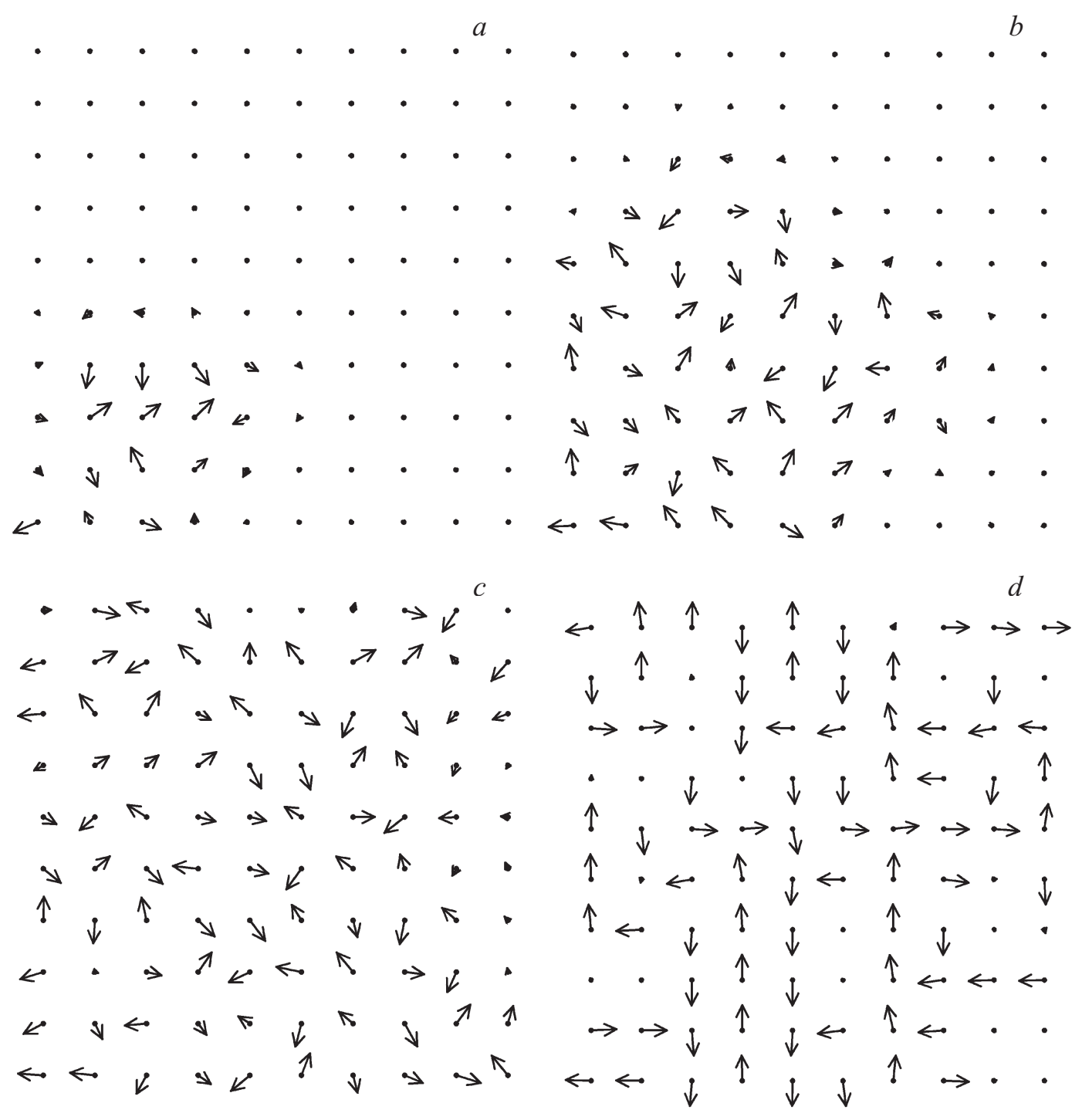

Рис. 5. Конфигурации, отражающие процесс распространения колебаний от углового диполя на всю систему в моменты времени $\tau=300,320,380(a-c)$ и равновесное состояние при $\tau \geq 1200(d)$.

$h_{0 x}=0.1(a)$ и $h_{0 x}=0.2(b-d)$. В случае $(a)$ после действия импульса система приходит к исходной конфигурации. В случае $(b)$ действие импульса приводит к переориентации выделенного углового диполя - в конечной конфигурации он ориентирован по оси $Z$ (остальные диполи остаются ориентированными по оси $X$ ). В случае $(d)$ угловой диполь в конечной конфигурации оказывается ориентированным по оси $X$ в отрицательном ее направлении. В случае же $(c)$ действие импульса приводит к тому, что динамика хаотической динамики распространяется от углового диполя на всю систему.

На рис. 5 приведены конфигурации, отражающие процесс распространения колебаний от углового диполя на всю систему (см. рис 4,c). Конфигурациям $(a-c)$ отвечают неравновесные состояния системы (различные фазы ее отклика на импульс поля) в моменты времени $\tau=300,320,380$. Конфигурации $(d)$ отвечает конечное равновесное состояние (при $\tau \geq 1200$ ), в котором дипо- ли ориентировались по направлению кристаллографических осей хаотическим образом в отношении дальнего порядка и сообразно действию диполь-дипольного взаимодействия в отношении ближнего порядка, (формируя тем самым блоки различного размера).

Рассмотрим также процесс перемагничивания решетки с конфигурацией рис. 5, $d$ импульсом магнитного поля, нарастающего и через интервал времени $\tau_{1}$ убывающего по экспоненциальному закону:

$\mathbf{h}(\tau)=\mathbf{h}_{0}\left\{\begin{array}{ll}1-\exp \left(-\tau / \tau_{0}\right), & \tau<\tau_{1} \\ {\left[1-\exp \left(\tau_{1} / \tau_{0}\right)\right] \exp \left(-\left(\tau-\tau_{1}\right) / \tau_{0}\right),} & \tau \geq \tau_{1} .\end{array}\right.$,

где $\tau_{0}-$ характерное время нарастания и убывания импульса. На рис. 6 приведена зависимость от времени компоненты $M_{x}$ магнитного момента системы при ее перемагничивании из исходного состояния в новую 


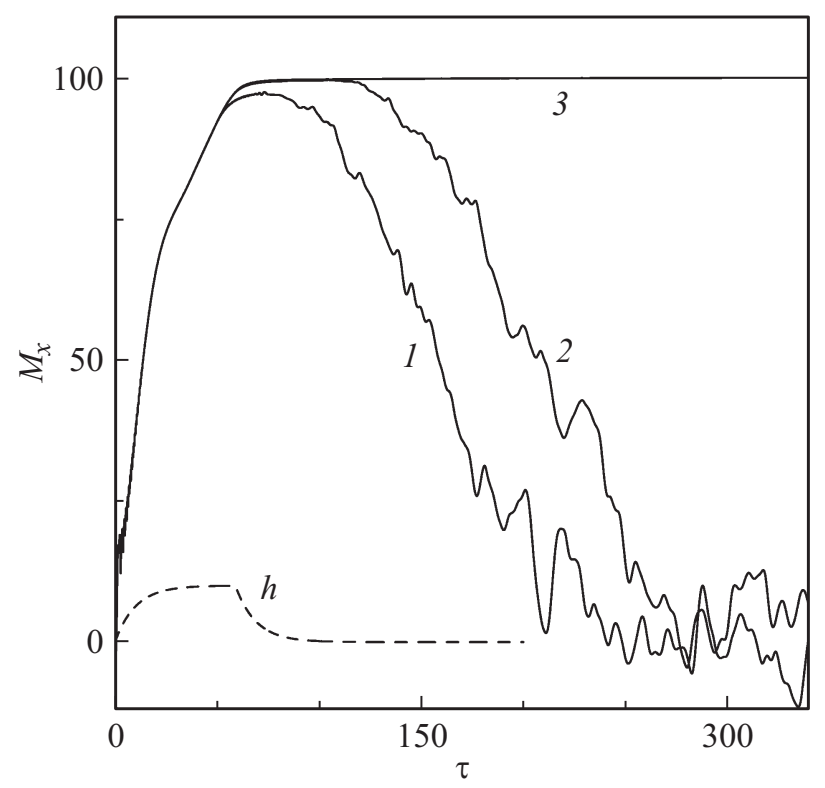

Рис. 6. Зависимость от времени компоненты $M_{x}$ магнитного момента системы при перемагничивании конфигурации (рис. 5, $d$ ) импульсом поля, поляризованным по оси $X$ с параметрами $h_{0 x}=10, \tau_{0}=10, \tau_{1}=50,58,59$ (кривые 1-3). Приведен также профиль импульса при $\tau_{1}=59$.

однородную конфигурацию импульсом магнитного поля, линейно поляризованным по оси $X$. Параметры импульса поля приняты следующими: $h_{0 x}=10, \tau_{0}=10$, $\tau_{1}=50,58,59$ (кривые $1-3$ ). На рисунке также приведен профиль импульса внешнего поля при $\tau_{1}=59$. Видно, что при данной амплитуде поля установление однородной конфигурации, ориентированной перпендикулярно плоскости системы, наступает только при длительности импульса $\tau_{1} \geq 59$. Увеличение амплитуды импульса позволяет уменьшить требуемую его продолжительность.

\section{5. Заключение}

Исследование дипольных решеток при наличии КА показало, что при расстоянии между ближайшими наночастицами $a / d \geq 2.7$ диполь-дипольное взаимодействие слабо влияет на ориентацию магнитных моментов диполей системы. В результате этого могут быть peaлизованы (в частности, с помощью локальных полей) различные равновесные конфигурации, при которых отдельные моменты ориентируются вдоль кристаллографических осей (,легких“ направлений). Вблизи границы устойчивого равновесия (при $2.7 \leq a / d \leq 2.8$ ) однородная конфигурация системы является чувствительной к колебаниям отдельных ее элементов. В частности, при ориентации 1-4 краевых диполей в плоскости решетки, а остальных диполей системы в перпендикулярной плоскости, воздействие импульсом на первые приводит к распространению хаотических колебаний на всю систему и установлению новой случайной по ориентации диполей конфигурации.
В результате реализуется большой по амплитуде отклик дипольной решетки, продолжительность которого в несколько раз превосходит длительность внешнего импульса. Амплитуда данного отклика меньше амплитуды отклика в случае, когда импульс резонансного поля эффективно действует на все диполи решетки. Однако рассматриваемая динамика слабо зависит от частоты внешнего поля в относительно больших интервалах ее значений, а также от других начальных условий. Это связано с тем, что отклик системы на внешний импульс формируется распространением колебаний на всю систему и последующими хаотическими колебаниями. Отсюда следует, что при увеличении числа элементов решетки будет увеличиваться продолжительность рассматриваемого отклика и его амплитуда. Показано также, что при определенных параметрах действующего импульса происходит не хаотизация всей системы, а переориентация отдельного ее элемента, лежащего в исходном состоянии в плоскости решетки.

\section{Список литературы}

[1] С.А. Гусев, Ю.Н. Ноздрин, М.В. Сапожников, А.А. Фраерман. УФН 170, 331 (2000).

[2] J.G. Zhu, Y. Zheng, G.A. Prinz. J. Appl. Phys. 87, 9, 6668 (2000).

[3] R. Skomski. J. Phys.: Condens. Matter. 15, R841 (2003).

[4] А.Ю. Галкин, Б.А. Иванов, А.Ю. Меркулов. ЖЭТФ 128, 1260 (2005).

[5] C. Chappert, A. Fert, F. Nguyen V. Dau. Nature Mater. 6, 813 (2007).

[6] Y.J. Chen, T.L. Huang, S.H. Leong, S.B. Hu, K.W. Ng, Z.M. Yuan, B.Y. Zong, B. Liu, V. Ng. Appl. Phys. Lett. 93, 10, 102 501-1 (2008).

[7] В.Л. Миронов, О.Л. Ермолаева, Е.В. Скороходов, J. Blackman. Изв. РАН 77, 37 (2013).

[8] П.В. Бондаренко, А.Ю. Галкин, Б.А. Иванов. ЖЭТФ 139, 1127 (2011).

[9] А.М. Шутый. ЖЭТФ 145, 1048 (2014).

[10] А.М. Шутый, Д.И. Семенцов. Письма в ЖЭТФ 99, 806 (2014).

[11] A.M. Shuty̌̆, S.V. Eliseeva, D.I. Sementsov. Phys. Rev. B 91, 024421 (2015).

[12] S. Khizroev, D. Litvinov. Perpendicular magnetic recording. Kluwer, N. Y-Boston-Dodrecht-London-Moscow (2004).

[13] Л.Н. Котов, Л.С. Носов, Ф.Ф. Асадуллин. ЖТФ 78, 60 (2008).

[14] N. Eibagi, J.J. Kan, F.E. Spada, E.E. Fullerton. IEEE Magn. Lett. 3, 4500204 (2012).

[15] Е.3. Мейлихов, Р.М. Фарзетдинова. ЖЭТФ 121, 875 (2002).

[16] Е.З. Мейлихов, Р.М. Фарзетдинова. ФТТ 56, 2326 (2014).

[17] А.М. Шутый, Д.И. Семенцов. ФТТ 59, 1703 (2017).

[18] А.Г. Гуревич, Г.А. Мелков. Магнитные колебания и волны. Наука, М. (1994). 